\title{
Sesame oleosins are minor allergens
}

\author{
Anna M. Ehlers ${ }^{1,2^{*}} \mathbb{D}$, Madlen Rossnagel ${ }^{3}$, Bettina Brix ${ }^{3}$, Mark A. Blankestijn ${ }^{1,2}$, Thuy-My Le $^{1,2}$, Waltraud Suer $^{3}$, \\ Henny G. Otten ${ }^{1}$ and André C. Knulst ${ }^{1,2}$
}

\begin{abstract}
Background: In daily practice, one-third of sesame allergic patients, confirmed by clinical history or food challenge, do not show any detectable specific IgE using current diagnostics. Currently used sesame extracts are waterbased and therefore lacking hydrophobic proteins like oleosins. Oleosins, the stabilizer of lipid droplets in plants, are described as allergens in sesame, peanut and hazelnut. In this study, we examine the role of oleosins in sesame allergy and their potential cross-reactivity between sesame and (pea)nuts.

Methods: Specific lgE and IgG sensitisation to native and heterologously expressed sesame components and oleosins from other nuts, free of seed storage proteins, was assessed by line blot and sera from 17 sesame allergic patients without detectable specific lgE sensitisation to sesame, and compared to 18 sesame allergic and 13 tolerant patients with specific IgE sensitisation to sesame.
\end{abstract}

Results: Sesame allergic patients without sensitisation showed no specific lgE to the tested sesame oleosins or components. Low levels of specific lgE to sesame oleosins were detected in 17\% of sesame allergic and $15 \%$ of tolerant patients with slgE sensitisation. Oleosins were recognised by serum IgG from multiple patients confirming immune reactivity and excluding technical issues leading to lack of specific lgE-binding to oleosins.

Conclusion: Sesame oleosins are minor allergens and appear to have no additonal value in diagnosing sesame allergy in adults based on slgE and slgG detection. There is a high need for additional diagnostic tools in those patients to minimize the number of required food challenges.

Keywords: Diagnostics, Food allergy, IgE hypersensitivity3 oleosins, Sesame

\section{To the editor}

Diagnosis of sesame allergy by measuring specific IgE (sIgE) is based on extracts or the major allergen: $2 \mathrm{~S}$ albu$\min$ Ses i 1 . However, this leads to false negative results in around $30 \%$ of sesame allergic patients, a high frequency compared to other food allergies [1]. To overcome this obstacle, we evaluated the role of oleosins in sesame allergy. Oleosins are oil-body stabilizing proteins and might be lacking in water-based extracts due to its hydrophobicity [2].

Adult sesame allergic and tolerant but sensitised patients who visited the outpatient clinic of the University Medical Centre Utrecht, The Netherlands, were retrospectively selected and allergy $(n=35)$ or tolerance

\footnotetext{
*Correspondence: a.m.ehlers@umcutrecht.nl

${ }^{1}$ Laboratory of Translational Immunology, University Medical Centre

Utrecht, Utrecht University, Utrecht, The Netherlands

Full list of author information is available at the end of the article
}

( $\mathrm{n}=13)$ was confirmed by food challenge or an experienced physician diagnosis. Sesame allergic patients were subdivided into patients without and with detectable sIgE (ImmunoCAP sesame extract $\geq 0.35 \mathrm{kU} / \mathrm{L}$; without $\mathrm{n}=17$; with $\mathrm{n}=18$ ). Patient characteristics are described in Table 1. Sera with sIgE against native and heterologously expressed oleosins from different nuts and seeds acted as positive controls. Ethical approval was acquired from the biobank committee of the University Medical Centre Utrecht, number 18-428.

Known sesame components and oleosins (from sesame, walnut, hazelnut, peanut and soy) with and without the hydrophobic domain based on the TMHMM model (prediction of transmembrane helices being not available for antibody binding) were heterologously expressed [3]. For comparisons to the native form, oil-body associated proteins (OAPs) were isolated from sesame, walnut and pecan using a modified previously described method 
Table 1 Patient demography of included sesame allergic and tolerant patients; allergy was defined by food challenge or an experienced physician

\begin{tabular}{|c|c|c|c|c|}
\hline & Group 1a $(n=17)^{a}$ & Group $1 b(n=18)^{a}$ & Group $2(n=13)^{a}$ & $p$ values \\
\hline Age (median) & $53(29-85)$ & $35(27-51)$ & $32(27-58)$ & 0.001 \\
\hline Sex female & $14(82 \%)$ & $10(56 \%)$ & $10(77 \%)$ & 0.1865 \\
\hline Food challenge & $8(47 \%)$ & $1(6 \%)$ & $1(8 \%)$ & 0.0034 \\
\hline Total lgE (kU/l) & 295 (39 to > 5000) & $4470(1010$ to $>5000)$ & > 5000 (518 to > 5000) & $<0.0001$ \\
\hline \multicolumn{5}{|l|}{ Symptoms ${ }^{\mathrm{b}}$} \\
\hline Mild (Müller 0) & $6(35 \%)$ & $6(33 \%)$ & N.A. & 0.9693 \\
\hline Moderate (Müller 1 +2) & $6(35 \%)$ & $6(33 \%)$ & N.A. & \\
\hline Severe (Müller 3+4) & $5(29 \%)$ & $6(33 \%)$ & N.A. & \\
\hline \multicolumn{5}{|l|}{ IgE measurements ${ }^{c}$} \\
\hline Sesame extract (n; median, range) & $17(0,0-0.32 \mathrm{kU} / \mathrm{L})$ & $18(4.5,0.5-75$ kU/L) & $13(3.9,0.4-48$ kU/L) & 0.5338 \\
\hline Ses i 1 ISAC (n; median, range) & $6(0$ ISU) & $10(2.9,0-22.5 \mathrm{ISU})$ & $3(0,0-6 \mathrm{ISU})$ & 0.4336 \\
\hline
\end{tabular}

${ }^{a}$ G1a sesame allergic patients without detectable slgE sensitisation, G1b sesame allergic patients with slgE sensitisation, G2 sesame tolerant patients with slgE sensitisation

b Symptom distribution of each group is shown in Additional file 3 :Figure S2

c ImmunoCAP and ISAC data were compared between group $1 \mathrm{~b}$ and 2 since group 1 a was selected by lacking slgE sensitisation; CAP $>0.35 \mathrm{kU} / \mathrm{L}$ was considered as positive

with an additional hydrophobic interaction chromatography (HIC) instead of a preparative gel electrophoresis to separate traces of seed storage proteins with similar molecular masses [4]. To examine the absence of seed storage proteins, enclosed proteins were identified by mass spectrometry and detected by western blot with anti-human IgE-alkaline phosphatase (AP). Sensitisation to these components was deeply investigated by measuring SIgE and sIgG levels using line blots (EUROLINE, EUROIMMUN, Luebeck, Germany) according to manufacturer's instructions. IgG subtypes were examined using an ELISA coated with different heterologously expressed oleosins (Ses i 4, Ses i Oleosin, Jug r Oleosin-1, Jug r Oleosin-2) and detected with anti-human IgG1-4 AP-conjugates. Detailed description of the methods is listed in Additional file 1.

Overall, the median age was 37 and allergic subjects suffered from typical symptoms being in line with an IgEmediated hypersensitivity (characteristics are shown in Table 1). Gastro-intestinal symptoms were always accompanied by OAS or skin reactions (Additional file 2: Figure S1) and the allergic reactions took place within several minutes to half an hour after ingestion (data from 8/35), supporting the diagnosis 'food allergy'.

The applied sesame OAPs fraction (pool of HIC-fractions 4 and 5) was free of seed storage protein traces as confirmed by western blot analysis with sera positive for these proteins from the study cohort (Fig. 1, part a). Before performing a HIC, these sera reacted with proteins around $15 \mathrm{kDa}$, similar molecular masses as oleosins. After the additional purification step, only the positive control (PC) still recognised proteins at this height. Additionally, no seed storage proteins but sesame oleosins were detected by mass spectrometry (Additional files 3 and 4: Figure S2 and Tables S1-S3).

Specific IgE-binding to sesame oleosins with levels above the detection limit (EAST-class 1-2) was detected in $17 \%$ of sensitised allergic and $15 \%$ of tolerant patients. However, none of the non-sensitised sesame allergic patients showed sIgE-binding neither to sesame oleosins nor to any other sesame components, except one serum showing low sIgE level to the 11S globulin Ses i 7 (G1a_6). Control sera, selected for sIgE binding to sesame (PC) or peanut oleosins (C1-3), showed sIgE binding to oleosins from different sources, confirming the binding capacity of heterologously expressed and native oleosins. Specific IgE-binding to oleosins in sensitised allergic patients was accompanied by recognising other sesame components, especially Ses i 1 and 2 whilst in tolerant patients was not (Fig. 1, part b and c). Patients showing IgE reactivity to heterologously expressed sesame oleosins were co-sensitised to heterologously expressed oleosins from walnut, hazelnut and peanut, indicating potential cross-reactivity (G1b_15, G1b_17). All oleosins expressed as full-length variant were recognised at least by one serum, except Cor a 12. In case of Cor a 13, the full-length and the hpvariant of Cor a 13 was recognised in parallel (G1b_17, PC).

Although oleosins were infrequently recognised by sIgE, they were bound by serum IgG from multiple patients across all groups confirming immune reactivity and excluding technical issues leading to lack of specific IgE-binding. Depletion of IgG by protein G columns in these sera did not result in SIgE binding (Additional file 5: 

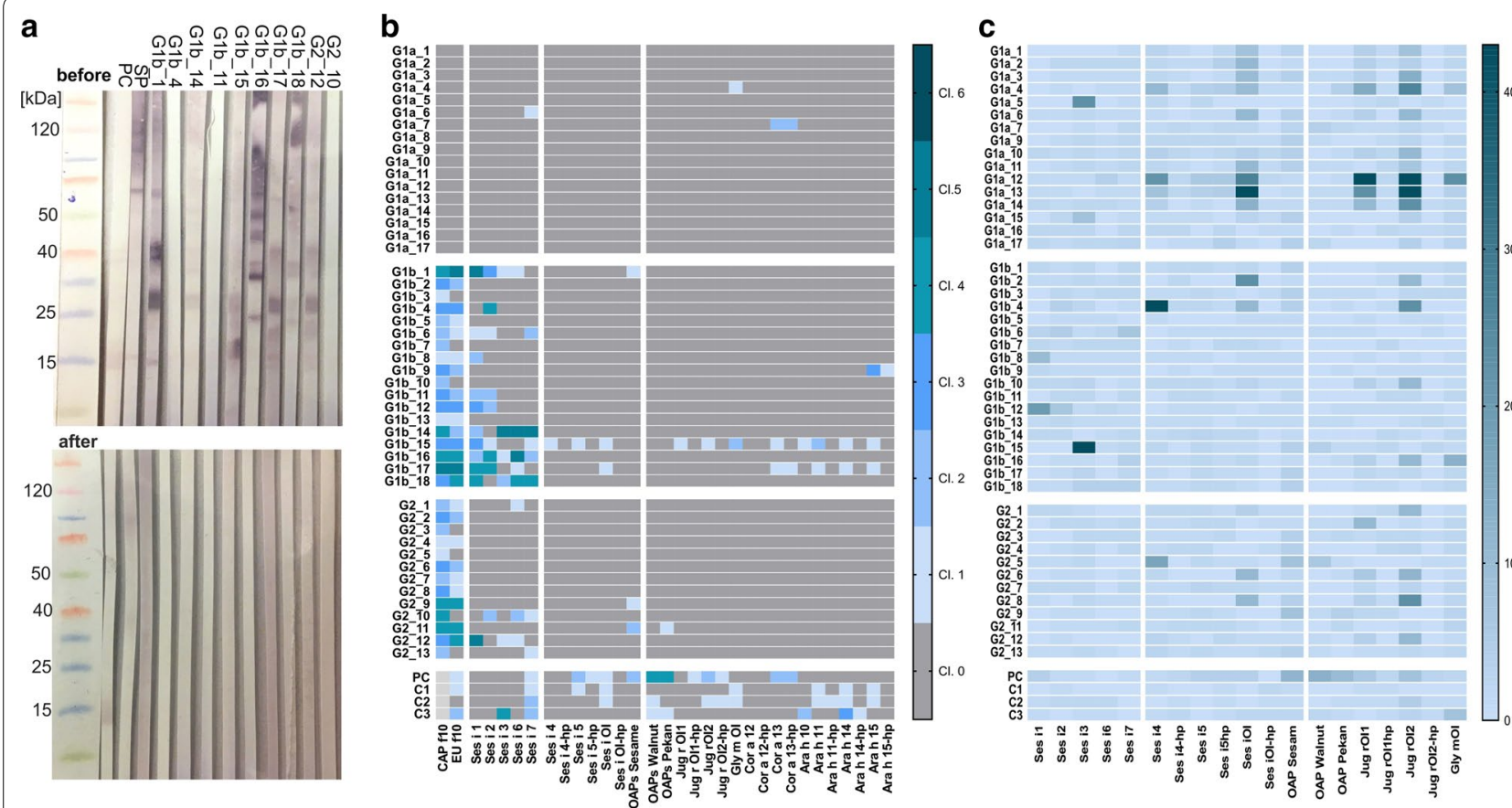

Fig. 1 Western blot analysis of native sesame OAPs fraction to confirm the absence of seed storage protein traces (a) and the specific lgE (b) and IgG sensitisation pattern (c) of all included patients. a Western-Blot analysis of an intermediate purification step (before HIC) and of the final OAPs fraction (after HIC) with sera containing slgE to sesame seed storage proteins and oleosins. Samples were separated by SDS-PAGE using a 4-12\% Bis-Tris gel and blotted onto a nitrocellulose membrane. Bound lgE were detected by anti-human IgE antibodies conjugate labelled with alkaline phosphatase; PC is a oleosin-positive serum, SP is a sesame seed storage positive serum not used in this study, sera G1 b_1, 4, 14, 11, 15-18, G2_12 and 10 are sera with specific lgE to sesame seed storage proteins and partly with slightly elevated slgE levels to sesame oleosins. b lgE sensitisation pattern to sesame extract from ImmunoCAP and EUROLINE, sesame components and oleosins from sesame, walnut, soy, hazelnut and peanut expressed as EAST (Enzyme-Allergo-Sorbent Test classification)-classes and sorted by severity of symptoms to sesame, light grey: not determined. c lgG-sensitisation pattern to sesame components and oleosins from sesame, walnut, pecan and soy expressed as EUROLINE (EL)-intensities and sorted by severity of symptoms to sesame. Gla sesame allergic patients without detectable slgE sensitisation, Glb sesame allergic patients with slgE sensitisation, G2 sesame tolerant patients with slgE sensitisation, $P C$ positive control selected by slgE to sesame oleosins, C1-3 positive controls selected by slgE to peanut oleosins

Figure S3), excluding competition between IgG and IgE. Specific IgG was mainly detected for Ses i 4, Ses i Ol, Jug r Ol-1 and Jug r Ol-2 and significantly increased in sesame allergic patients without detectable sIgE sensitisation compared with the other groups (Ses i 4: G1a vs. G1b, $p=0.0014$; Ses i Ol: G1a vs. G2, $p=0.03$; Jug r Ol-2: G1a vs. G1b, $p=0.0049$ ). The most prevalent subtype recognising oleosins was IgG1, while IgG2, IgG3 and IgG4 levels were dependent on the oleosin of interest (Additional file 6 and 7: Figures S4 and S5).

Up to $30 \%$ of sesame allergic patients, including those suffering from severe reactions, cannot be diagnosed by commercially available diagnostic tests $[1,5]$. Launched aqueous sesame extracts do not contain hydrophobic proteins like oleosins. We demonstrated that oleosins were clearly recognized in $17 \%$ of sensitised sesame allergic patients, but did not have any additional diagnostic value compared to sesame extract and Ses i 1, especially in patients without detectable IgE sensitisation.
Contrary to our findings, $90 \%$ recognition in sesame allergic patients (31\% without sIgE to sesame extract) was reported previously [6]. Moreover, OAPs reactivity was detected in $36 \%$ of hazelnut allergic patients and therefore considered relevant diagnostic markers, particularly in patients without detectable sIgE [7]. This discrepancy might be explained by the absence of seed storage proteins or fragments thereof [8] in our native preparation accomplished by HIC which was confirmed by western blot analysis and mass spectrometry.

Oleosins were recognised by sIgE levels slightly above the detection limit (EAST-class 1-2). This is in line with a hazelnut study across Europe showing a prevalence of $20 \%$ for the hazelnut oleosin Cor a 12, but low SIgE titre up to $1 \mathrm{kU} / \mathrm{l}$ ( $\triangleq$ EAST-class 2) [9]. In our test system, even lower sIgE titres might be explained by competition between different oleosins coated on the same line blot.

In conclusion, sesame oleosins are minor allergens and appear to have no additional value in diagnosing sesame 
allergy in adults based on sIgE and sIgG detection. We propose a prospective study to evaluate the diagnostic value of direct basophil activation tests due to the high need for additional diagnostic tools in those patients.

\section{Additional files}

Additional file 1. Supplementary methods. Detailed description of the methods.

Additional file 2: Figure S1. Distinct symptom distribution between sesame allergic patients with and without detectable slgE sensitisation. Sesame allergic patients without detectable slgE sensitisation (G1a) showed more often skin related reactions (Mueller 1) compared to patients with sensitisation (G1b). The other way around, patients of $\mathrm{G} 1 \mathrm{~b}$ showed more often gastro-intestinal symptoms (Mueller 2) and cardiovascular reactions (Mueller 4).

Additional file 3: Figure S2. Gel images for mass spectrometry analyses of OAPs fractions. Gel images of elution fractions of OAPs from sesame, walnut and pecan nut after hydrophobic interaction chromatography used for mass spectrometry analyses.

Additional file 4: Table S1-S3. Enclosed proteins in native OAPs fractions. The native OAPs fractions were analysed by mass spectrometry. Table 1-3 show the identified proteins in those fractions.

Additional file 5: Figure S3. IgG depletion does not influence the recognition of sesame oleosins by slgE. After IgG depletion, sera with high slgG levels to oleosins (G1a_4, G1a_12, G1a_13 and G2_8) showed no increase in slgE levels to sesame components or oleosins while sera with low slgG levels but IgE sensitisation to sesame (G1b_1, G1b_15, G1b_17, G2 9 and G2_11) showed scarcely a decrease in slgE levels, confirming no depletion of $\mathrm{lgE}$ during $\mathrm{lg} G$ depletion. One serum positive for sesame oleosins, G1b_15, displayed an increase in slgE levels to oleosins although no slgG to these components were detected.

Additional file 6: Figure S4. Subtype analysis of sera with elevated slgG levels for Ses i 4, Ses i Ol, Jug r Ol-1 and Jug r Ol-2. Detection of the lgG subtype bound to Ses i 4, Ses i Oleosin, Jug r Oleosin-1 and Jug r Oleosin-2 from serum with specific $\lg G$ to oleosins (EUROLINE-intensities $>$ 8); Scatter-blot with the measured OD value ratios (sample/negative value [NV]) divided by subtype for each oleosin. Horizontal lines mark the mean value. Green: Group 1a - sesame allergic patients without detectable slgE sensitisation; Red: Group 1b - sesame allergic patients with slgE sensitisation; Black: Group 2 - sesame tolerant patients with slgE sensitisation.

Additional file 7: Figure S5. Comparison of lgG subtype between allergic and tolerant patients. Detection of the lgG subtype bound to Ses i 4, Ses i Oleosin, Jug r Oleosin-1 and Jug r Oleosin-2 from serum with specific lgG to oleosins (EUROLINE-intensities $>8$ ); the data were separated by allergic and tolerant patients, lgG subtype and protein (oleosin).

\section{Abbreviations}

EAST: Enzyme-Allergo-Sorbent Test; EL: EUROLINE; HIC: hydrophobic interaction chromatography; hp-variant: heterologous expressed oleosin without hydrophobic domain; NCBI: National Centre of Biotechnology Information; OAPs: oil-body associated proteins; slgE: specific lgE; SPT: skin prick test; TMHMM: transmembrane prediction by hidden Markov model.

\section{Acknowledgements}

We would like to thank M. Klug and S. Brandhoff for technical assistance, Y. Weimann for fruitful discussions and Y. Denno for performing the mass spectrometry analyses. Line blots and corresponding reagents were kindly provided by EUROIMMUN AG, Lübeck, Germany.

\section{Authors' contributions}

$A E, M R$, WS, HO designed the experiments; $A E, M B, T M L$, AK selected the retrospective patient cohort; $A E$, MR performed the experimental procedures; $A E$ performed data collection and analyses and drafted the manuscript; $M R, B B$, $M B, T M L, W S, H O, A C$ contributed to data interpretation and critically revised the manuscript. EUROIMMUN was not involved in the overall design of the study. All authors read and approved the final manuscript.

\section{Funding}

This project was partly financially supported by EUROIMMUN AG, Lübeck, Germany.

\section{Availability of data and materials}

All data generated or analysed during this study are included in this published article and its Additional information files.

\section{Ethics approval and consent to participate}

This study was carried out in accordance with the University Medical Centre Utrecht, Biobank Regulations, which are in compliance with the applicable national and international laws and regulations. These regulations permit the use of 'residual material from diagnostic testing' for research, unless the patient objects (Article 8,'no objection' procedure). None of the included patients objected the use of their serum. The protocol was approved by the Biobank Research Ethics Committee of the University Medical Centre Utrecht under the protocol number 18-428.

\section{Consent for publication}

Not applicable.

\section{Competing interests}

B. Brix, M. Rossnagel and W. Suer are employees of EUROIMMUN. The research position of A. Ehlers is partially funded by EUROIMMUN. Other authors have no competing interests to declare.

\section{Author details}

${ }^{1}$ Laboratory of Translational Immunology, University Medical Centre Utrecht, Utrecht University, Utrecht, The Netherlands. ${ }^{2}$ Department of Dermatology and Allergology, University Medical Centre Utrecht, Utrecht University, Utrecht, The Netherlands. ${ }^{3}$ EUROIMMUN AG, Lübeck, Germany.

Received: 5 February 2019 Accepted: 20 June 2019

Published online: 28 June 2019

\section{References}

1. Permaul P, Stutius LM, Sheehan WJ, Rangsithienchai P, Walter JE, Twarog FJ, et al. Sesame allergy: role of specific lgE and skin-prick testing in predicting food challenge results. Allergy Asthma Proc. 2009;30(6):643-8.

2. Tzen JT, Peng CC, Cheng DJ, Chen EC, Chiu JM. A new method for seed oil body purification and examination of oil body integrity following germination. J Biochem. 1997;121(4):762-8.

3. Sonnhammer EL, von Heijne G, Krogh A. A hidden Markov model for predicting transmembrane helices in protein sequences. Proc Int Conf Intell Syst Mol Biol. 1998;6:175-82.

4. Schwager C, Kull S, Krause S, Schocker F, Petersen A, Becker WM, et al. Development of a novel strategy to isolate lipophilic allergens (oleosins) from peanuts. PLoS ONE. 2015;10(4):e0123419.

5. Derby CJ, Gowland MH, Hourihane JO. Sesame allergy in Britain: a questionnaire survey of members of the Anaphylaxis Campaign. Pediatr Allergy Immunol. 2005;16(2):171-5.

6. Leduc V, Moneret-Vautrin DA, Tzen JT, Morisset M, Guerin L, Kanny G. Identification of oleosins as major allergens in sesame seed allergic patients. Allergy. 2006;61(3):349-56.

7. Zuidmeer-Jongejan L, Fernández-Rivas M, Winter MG, Akkerdaas JH, Summers C, Lebens A, et al. Oil body-associated hazelnut allergens including oleosins are underrepresented in diagnostic extracts but associated with severe symptoms. Clin Transl Allergy. 2014;4(1):4. https:// doi.org/10.1186/2045-7022-4-4. 
8. Koppelman SJ, Knol EF, Vlooswijk RA, Wensing M, Knulst AC, Hefle SL, et al. Peanut allergen Ara $\mathrm{h}$ 3: isolation from peanuts and biochemical characterization. Allergy. 2003;58(11):1144-51.

9. Datema MR, Zuidmeer-Jongejan L, Asero R, Barreales L, Belohlavkova $\mathrm{S}$, de Blay F, et al. Hazelnut allergy across Europe dissected molecularly: a EuroPrevall outpatient clinic survey. J Allergy Clin Immunol. 2015;136(2):382-91.

\section{Publisher's Note}

Springer Nature remains neutral with regard to jurisdictional claims in published maps and institutional affiliations.
Ready to submit your research? Choose BMC and benefit from:

- fast, convenient online submission

- thorough peer review by experienced researchers in your field

- rapid publication on acceptance

- support for research data, including large and complex data types

- gold Open Access which fosters wider collaboration and increased citations

- maximum visibility for your research: over $100 \mathrm{M}$ website views per year

At BMC, research is always in progress.

Learn more biomedcentral.com/submissions 